\title{
Information for action: AJLM's special issue on transforming the quality of laboratory medicine through the Strengthening Laboratory Management Toward Accreditation programme
}

This inaugural special issue of the African Journal of Laboratory Medicine spotlights the critical need for continuous quality improvement in laboratories and the impressive global expansion and impact of one programme aimed to make such improvements a reality: the Strengthening Laboratory Management Toward Accreditation (SLMTA) programme.

SLMTA that teaches laboratory managers how to implement practical quality management systems in resource-limited settings using available resources. With a series of short courses and work-based improvement projects supported by site visits and mentoring, SLMTA is designed to achieve rapid, measureable improvement in laboratories. SLMTA was launched in 2009, and has since been implemented in some 617 laboratories in 47 countries worldwide.

This special issue provides a comprehensive collection of programme results, including a detailed description of the SLMTA methodology and variations thereof, analyses of global programme data, and a summary of the progress made in developing a cadre of indigenous SLMTA trainers to facilitate global scale-up. A two-part comprehensive review of the literature summarizes the qualitative and quantitative results, and identifies strategic directions for the future of the programme.

Equally, or perhaps more important, are the numerous reports of country- and laboratory-level experiences providing in-depth lessons learned from ground-level implementers. From these papers we learn about factors contributing to success in implementing a quality management system, as well as pitfalls to beware of, and glean practical insights from the SLMTA pioneers who have gained first-hand experience striving for continuous quality improvement. These articles focus on navigating the balance between country ownership and effective partnership, using innovative incentives to accelerate improvements, building local human resources, and developing a culture of quality in laboratories that is sustainable. Other articles relate experiences, such as continuing on to the goal of international accreditation, and addressing the need to extend the SLMTA programme beyond the laboratory to all parts of the health system.

The remaining articles focus on specific facets of the programme, including the impact of mentorship, whether establishing in-country training facilitators is less expensive than using international trainers, and the pros and cons of conducting centralized versus facility-based training. Others explore how to engage local resources, such as research laboratories and partners, and the benefit of using electronic tools to streamline the audit process.

Until now, little has been published on this groundbreaking programme, as efforts thus far have been focused on implementation rather than evaluation. This collection contains a vast wealth of information from a programmatic and observational point of view. The more complicated work of rigorous programme evaluation - for example, studies using randomized interventions and control groups; formal calculations of cost-effectiveness; and assessment of the health impact of laboratory quality improvement - remains to be done.

By combining these studies into a single collection, we hope to assist readers with assimilation of the results into a meaningful understanding of the SLMTA programme and its implementation. Whether used by individual laboratories to improve quality of services, by country-level managers and partners to guide SLMTA implementation, or by Ministry of Health leaders and other decision-makers as a source of evidence for large-scale planning, the data and insights of those who have successfully implemented the programme provide a wealth of knowledge and information for evidence-based decision-making to ensure continuous quality improvement for better patient care and public health outcomes.

The Supplement Coordinators and Guest Editors

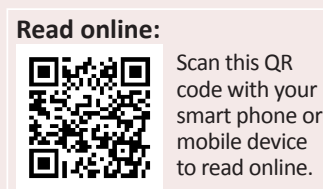

How to cite: The Supplement Coordinators and Guest Editors. Information for action: AJLM's special issue on transforming the quality of laboratory medicine through the Strengthening Laboratory Management Toward Accreditation programme. Afr J Lab Med. 2014;3(2), Art. \#279, 1 page. http://dx.doi.org/10.4102/ajlm.v3i2.279 